\title{
República y concordia. Política y paz en Agustín de Hipona*
}

\author{
Diana Marcela Sánchez Barbosa*
}

Recibido: 8 de agosto de 2015 - Aprobado: 10 de diciembre de 2015

\section{Resumen}

Se parte de la premisa de que para San Agustín la felicidad del ser humano está determinada por la paz, pues esta se constituye en uno de sus anhelos más profundos. Por ello, el artículo inicia con la exposición del proceso que va formando el pensamiento de este personaje, analizando en sus escritos la relación que desde la Antigüedad se ha presentado entre el tema de las instituciones sociales y políticas y la concepción religiosa de la realidad. Se muestra así como en los escritos agustinianos se percibe una respuesta concreta respecto a la pregunta sobre cómo ser cristiano y, al mismo tiempo, cómo pertenecer a una comunidad terrena y a un orden político. De esta forma, se desarrolla el concepto de orden en su pensamiento, para explicar la manera cómo la vida en comunidad y la República le ofrecen al hombre un camino que permite no solo el progreso de la sociedad, sino la consecución de la paz.

Palabras clave: San Agustín, concordia, Ciudad de Dios, orden, República.

Artículo de reflexión. Este trabajo presenta los resultados parciales de la investigación en torno a la herencia del pensamiento ciceroniano en la obra de Agustín, de manera particular, en el ámbito político. DOI: http://dx.doi.org/10.15332/ s0120-8454.2016.0089.04

* Magíster en Filosofía de la Pontificia Universidad Javeriana y licenciada en Filosofía de la misma Universidad. Actualmente se desempeña como docente de Filosofía en el Colegio Nueva Granada de Bogotá. Dirección postal: Carrera 2 Este \# 70-20, Bogotá, Colombia. Correo electrónico: diana.sanchez@javeriana.edu.co. 


\title{
Republic and harmony. Politics and peace in the work of Augustine of Hippo*
}

\author{
Diana Marcela Sánchez Barbosa**
}

\section{A bstract}

The premise that, for Augustine, happiness of human beings is determined by peace, as it constitutes one of their deepest desires, is the basis for this paper. Therefore, the process that builds the thought of this character is exposed and the relationship that, from ancient times, has arisen between social and political institutions and religious conception of reality is analyzed. This paper explains how to perceive, in the Augustinian writings, a definite answer regarding the question of how to be a Christian and, at the same time, belong to a mundane community and a political order. Thus, the concept of order develops in his mind to explain the way how community life and the Republic offer men a way that allows, not only the progress of society, but the pursuit of peace.

Keywords: Saint Augustine; Harmony; City of God; Order; Republic.

Reflection paper. This paper presents the preliminary results of the research on the inheritance of Ciceronian thought in the work of Augustine, particularly, on the political field. DOI: http://dx.doi.org/10.15332/s0120-8454.2016.0089.04

.. Master in Philosophy from Pontificia Universidad Javeriana and degree in Philosophy from the same University. Currently working as a Philosophy Teacher in Colegio Nueva Granada de Bogotá. Address: Carrera 2 Este \# 70-20, Bogota, Colombia. Email: diana.sanchez@javeriana.edu.co. 


\title{
République et concordance. Politique et paix chez Augustin d'Hippone*
}

\author{
Diana Marcela Sánchez Barbosa**
}

\section{Résumé}

On part de la prémisse que pour Saint Augustin le bonheur de l'être humain est déterminé par la paix, car celle-ci constitue l'un de ses souhaits les plus profonds. C'est pour cela que l'article commence avec la présentation du processus qui forme peu à peu la pensée de ce personnage, analysant dans ses écrits la relation qui s'est présentée depuis l'Antiquité entre le thème des institutions sociales et politiques et la conception religieuse de la réalité. On montre ainsi comment dans les écrits d'Augustin on perçoit une réponse concrète face à la question de comment être chrétien et, en même temps, comment appartenir à une communauté terrena et à un ordre politique. De cette manière, se développe le concept d'ordre dans sa pensée, pour expliquer la manière dont la vie en communauté et la République offrent à l'homme un chemin qui permet non seulement le progrès de la société, mais aussi l'obtention de la paix.

Mots clés: Saint Augustin, concordance, Ville de Dieu, ordre, République.

Article de réflexion. Ce travail présente les résultats partiels de la recherche autour de l'héritage de la pensée cicéronienne dans l'œuvre d'Augustin, de manière particulière, dans le domaine politique. DOI: http://dx.doi.org/10.15332/ s0120-8454.2016.0089.04

* Magister en Philosophie de l'Université Javeriana et licenciée en Philosophie de la même université. Actuellement elle travaille comme professeur de Philosophie à l'École Nueva Granada de Bogota. Adresse postale: Carrera 2 Este \# 70-20, Bogota, Colombie. E-mail: diana.sanchez@javeriana.edu.co. 
El anhelo de paz es natural en el hombre. Por eso, desde la Antigüedad se ha reflexionado sobre ella, no solo en tiempos de concordia y tranquilidad, sino en los de guerra y conflicto. Agustín no es la excepción, pues sabe que la felicidad del ser humano está determinada por la paz, y afirmará que, pese a la dificultad que supone alcanzarla, no existe nada que el hombre desee más.

El Hiponense se enfrenta a una situación particular a la hora de abordar dicha cuestión, pues por las Sagradas Escrituras, el cristiano sabe que pertenece a otro reino y, por lo tanto, su vida está en función de él. Sabe, además, que ese reino es el cumplimiento de la promesa de Cristo y que allí gozará de la visión eterna de Dios, que supone, asimismo, una vida bienaventurada en la eternidad. Sin embargo, esta es una promesa que se cumplirá al final de los tiempos, cuando llegue el día del juicio, en el que justos y pecadores alcanzarán, unos la vida y otros la muerte eterna. De este modo, el cristiano se enfrentará al problema de cómo asumir la vida en la Tierra mientras espera el día del juicio, ya que hasta cierto punto hará parte de dos sociedades opuestas, una que usa sin amar y otra que ama sin dejar de permanecer en la primera. En este caso, la razón exigiría que de estos dos partidos se eligiera uno u otro:

si los cristianos rehusaban cumplir con los sacrificios habituales y honrar a los que los presiden, entonces ellos no deben dejarse emancipar, ni casarse, ni criar hijos, ni cumplir ninguna de las obligaciones de la vida común [...] pero si contraen matrimonio, si tienen hijos, si participan de los gozos de la vida, así como de los males que le son inherentes, entonces deben ellos pagar tributo de honor a quienes velan por esas cosas, cumplir los deberes que impone la vida, hasta que sean libertados de los lazos terrestres. (Gilson, 1954, p. 34)

Podríamos preguntarnos entonces, qué hacer con el conflicto que se genera entre los hombres que no consideran nada más que la patria terrena y los cristianos, que son, ante todo, ciudadanos de la ciudad de Dios. Estos no tienen mayor interés en lo que tiene que ver con la Tierra, pues saben que están en ella solo de paso y nada de lo que se encuentra allí es comparable con lo que les ha sido prometido, pues aunque lo que hay en la Tierra es bueno, está inmerso en el desorden, es corruptible y hace parte de la dinámica del pecado.

Este es uno de los problemas a los que se enfrenta Agustín cuando escribe la Ciudad de Dios. Las circunstancias por las que pasaba el Imperio y las condiciones en las que se encontraban los cristianos hacen necesaria una respuesta a las cuestiones sobre a qué orden pertenece el pueblo de Dios, a qué sociedad, a qué reino debe guardar fidelidad, pero sobre todo, cómo debe hacer frente a las eventualidades propias de la vida mortal, sin perder el horizonte al que se debe dirigir en pro del cumplimiento de la promesa, esto es, cómo ser cristiano y, al mismo tiempo, hacer parte de un orden político y de las circunstancias de la vida en la Tierra.

Es bien conocido que los temas vinculados con la esfera política, tanto en el ámbito práctico como en el de la reflexión, escapan al interés de Agustín. Él 
siempre prefirió la vida tranquila, lejos de los afanes de la vida pública, y aquella en la que pudiera dedicarse al estudio y la contemplación de la verdad. Pese a esto, por diversas circunstancias, desde muy joven se involucró en grupos y comunidades de diversa índole. Los maniqueos son una de las comunidades a las que Agustín se afilió.

El maniqueísmo o religión de la luz era un movimiento de carácter religioso, fundado por Mani en el siglo iı d. C. Proveniente de Babilonia, Mani estaba seguro de que las enseñanzas de Buda, Zoroastro y Jesús constituían el verdadero mensaje de Dios. Como dicho mensaje estaba incompleto, la tarea de Mani sería la de compartir con el mundo la revelación completa y acabada. De este modo, combinando elementos del budismo, el zoroastrismo y el cristianismo, junto con algunas ideas gnósticas, Mani, con poco más de treinta años, comenzó a difundir el mensaje que le había sido revelado por primera vez a los doce años, y luego a los veinticuatro (Cf. Bermejo, 2008, p. 118).

Agustín encontró en el maniqueísmo una comunidad de amigos con la que se sentía a gusto, donde podía experimentar un modo de vida despreocupado y compatible con las labores que un maestro de retórica como él debía realizar. Hizo grandes amigos en la comunidad de los maniqueos; allí encontró otros jóvenes cultos, inteligentes y que compartían con él algunas de las inquietudes que lo aquejaban desde hacía ya mucho tiempo. Sin embargo, las enseñanzas de Mani no respondieron a las preguntas que el grupo de jóvenes tenía, y Agustín se fue desilusionando gradualmente de las enseñanzas del maniqueísmo. Luego de pertenecer por cerca de diez años a la comunidad, Agustín solo se mantuvo como miembro de la secta por las amistades que había cultivado, mas no por las verdades que encontró en la doctrina.

Luego de su paso por el maniqueísmo, y tras un periodo de profundos cambios, que incluyen su acercamiento a la Iglesia católica y a algunos de sus grandes líderes, Agustín toma la decisión de convertirse al cristianismo, sin ninguna reserva. Comenzó así una nueva vida, pero no en soledad; Agustín sabía que el nuevo camino debería emprenderlo de la mano de otros con la misma disposición. De este modo, conformó una pequeña comunidad con su madre, su hijo, su hermano y algunos de sus amigos más cercanos. El grupo viajaría a Casiciaco, cerca de Milán, pues aquel lugar les ofrecía un ambiente más tranquilo, que les permitiría tomar distancia de la algarabía de la gran ciudad y así, en la tranquilidad del campo, llevar a cabo el proyecto de vida que habían acordado, avanzar en sus indagaciones y, al mismo tiempo, cultivar la fe y la razón y prepararse para recibir el bautismo.

Agustín siempre participó con entusiasmo en los grupos a los que perteneció, pero solo cuando llegó a Hipona, buscando un lugar apacible para establecer su comunidad filosófica, se vio involucrado en la vida cívica y política de una gran ciudad.

Como obispo de Hipona, se ve obligado a participar activamente en la vida pública de la ciudad. Dentro de las funciones que debía desarrollar una persona 
con un cargo tan distinguido, están no solo las relacionadas con la predicación, la celebración de los sacramentos y las actividades de tipo pastoral, sino también las de orden judicial, ya que el Imperio había facultado a los obispos para arbitrar en los procesos civiles, dada su propia imposibilidad de llegar a todos los rincones de su extenso territorio.

Las tareas que desarrollaba como obispo, los sucesos que acontecieron en el Imperio, (sobre todo la toma de Roma por parte de Alarico y sus tropas, en el año 410), los conflictos que se vivieron al interior la Iglesia (como el cisma donatista) y las acusaciones de los paganos contra la religión cristiana (según las cuales, esta es la principal culpable de las circunstancias por las que pasaba el Imperio) hacen que Agustín se involucre y asuma decididamente tales problemáticas, siempre y ante todo como un cristiano comprometido, que se ve en la obligación de defender los intereses de la comunidad de fieles siervos de Dios, pues estas disputas trascienden el ámbito de las pugnas entre vecinos para convertirse en situaciones que con frecuencia afectan a la comunidad, pero en particular al pueblo de Dios y a la Iglesia misma.

Ahora bien, aunque las discusiones que Agustín entabla con distintos grupos de la Iglesia y de la comunidad, así como sus posiciones sobre el Gobierno, la vida en sociedad y las costumbres del pueblo cristiano, pueden seguirse tanto en su obra temprana como en la tardía, es en la Ciudad de Dios donde se encuentra un estudio más detallado de dichas reflexiones.

La Ciudad de Dios es uno de los escritos más importantes de Agustín; en él vuelve, entre otros, sobre la historia, la filosofía y la cultura del mundo antiguo, pero lejos de ser una síntesis de dichos elementos, la obra constituye la lectura que un cristiano del siglo v hace de su tiempo. Esta obra ha sido considerada por muchos una enciclopedia del mundo antiguo; aborda temáticas que, en el marco de la historia de la humanidad y la acción de Dios en ella, van desde la Creación y el final de los tiempos hasta la pertinencia de ciertos cultos religiosos, la clasificación de las principales sectas filosóficas y las relaciones entre ángeles, demonios y hombres. Sin embargo, la obra va más allá de ello, pues Agustín también asume la labor de disipar prejuicios, deshacer una serie de problemas mal planteados y mal resueltos y, sobre todo, establecer la posición del pueblo cristiano al respecto.

Una obra de tal magnitud, cuya composición tomó a su autor más de quince años, exige un lector que conozca, por un lado, ciertos aspectos del mundo antiguo - concretamente de la cultura latina - , que permitan situar al autor y a la obra en un contexto específico, y, por el otro, cuestiones básicas del pensamiento de Agustín, que aunque estén presentes en la obra, no lo están de manera explícita ni ampliamente expuesta. Ejemplo de ello son los temas del amor, la gracia y la Trinidad.

Más aún, es importante señalar que aunque se conozcan estos elementos, que podemos denominar teóricos, es necesario también conocer a Agustín, el hombre, 
pues las circunstancias vitales del autor están siempre implicadas en sus escritos. Por ejemplo, tener en cuenta que el mismo Agustín se reconoce casi siempre como teólogo, y pocas veces como filósofo, no solo posibilita una lectura más fiel del texto agustiniano, sino que permite comprender que sus investigaciones, aunque se realicen a la luz de la razón, siempre se fundamentan en la fe y encuentran en la Sagrada Escritura su máxima autoridad.

El elemento religioso no es exclusivo de los escritos agustinianos; en la Antigüedad grecolatina, la esfera religiosa es un factor intrínseco a los distintos ámbitos de la existencia, por eso Agustín vincula estrechamente la vida del Imperio, primero con los diferentes cultos paganos, y luego con la cristiandad. Este hecho resulta de particular importancia, pues indica-en nuestro casoque el tema de las instituciones sociales y políticas no puede desvincularse de una concepción religiosa de la realidad, y menos aún de los principios básicos del cristianismo, pues esto implicaría alejarnos no solo del contexto histórico particular, sino de la comprensión agustiniana de la realidad.

Ahora bien, el cristiano sabe que su reino, el de la promesa de Cristo, donde gozará de la bienaventuranza eterna, no pertenece a este mundo. Aquel es eterno, perfecto e inmutable, y allí los justos gozarán de la vida eterna luego del juicio final. Por el contrario, este mundo es finito, desordenado, mudable, y los hombres están sujetos a las determinaciones propias de esta condición. Esto genera un problema para el cristiano, y es el de cómo asumir la vida en la Tierra mientras espera el día del juicio, pues entre tanto hace parte de dos sociedades: la Ciudad de Dios y un reino terreno, por ejemplo, el Imperio romano. Es pertinente, entonces, preguntarse: ¿cómo vive el cristiano, que tiene puestos su mente y su corazón en el reino que ama, pero que, como peregrino en la Tierra, vive en el mundo de los hombres y tiene que participar de las dinámicas que le son propias?

El interés de Agustín por estos problemas no puede considerarse estrictamente intelectual, pues las circunstancias por las que pasaba el Imperio y las condiciones en las que se encontraba el pueblo cristiano pedían más una respuesta concreta a la pregunta sobre cómo ser cristiano y, al mismo tiempo, pertenecer a una comunidad terrena y a un orden político, que unas largas disertaciones de orden teórico. Para abordar este tema es necesario volver sobre algunos elementos que nos acerquen a la comprensión agustiniana de la realidad y, de manera concreta, del hombre y su dinámica en el mundo. Es necesario un fundamento metafísico, que nos proporcione los elementos mediante los cuales podamos acceder a una perspectiva general de la Creación en su relación con el Creador.

Aunque Agustín estudia detalladamente no solo el evento que constituye la Creación, sino la forma en que Dios se hace presente en ella, no volveremos sobre cada uno de los elementos que son objeto de su reflexión, sino que nos centraremos en tres: la medida, el número y el peso, que son las determinaciones fundamentales según las cuales Dios no solo creó el universo, sino que son aquellas con las que justísimamente lo dispone y ordena. 
El orden es el principio de conformidad con el que todo lo que existe fue creado, tiende a un fin y se sitúa en el lugar que le corresponde. Como todo se encuentra en el ámbito del orden, podemos decir que si algo no pertenece a él, simplemente no existe. En la Creación, Dios ordenó todas las cosas en medida, número y peso (Sabiduría, 11, 20); esta es la tríada de la que se sirve Agustín para describir la estructura fundamental de la Creación, lo que hay en ella y la dinámica que hay entre ella y el Creador. Brevemente podemos señalar que "la medida es la que determina el modo de existir de todo ser, por número, el que suministra la forma de la existencia y por peso, el que reduce a la estabilidad y quietud a todo ser" (Agustín, Gn.litt. Iv, 3, 7). Parafraseando a Agustín, basta levantar y extender los ojos de la mente a la universalidad de las cosas, y nada hallaremos que no esté ordenado y ocupando un lugar distinto y acomodado (De Ord. 2. 12. 11.). Agustín sitúa el concepto de orden dentro del marco especulativo cristiano $\mathrm{y}$, por lo tanto, gira en torno a la idea de un Dios creador, personal y providente; Dios, que por demás tiene la particularidad de ser trinitario, es decir, un mismo Dios, de idéntica sustancia, inseparable igualdad y unicidad en tres personas: Padre, Hijo y Espíritu Santo.

Aunque el término orden tiene carácter polisemántico, podemos decir, de manera general, que se refiere a la organización interna de la realidad, es siempre positivo, dinámico y se vincula con la unidad. Esta unidad de orden es ordenante, es decir, es la causa de la unificación de lo ordenado; lo ordenado, por su parte, es "el conjunto de realidades físicas, sus operaciones y sus interrelaciones" (Moreno, 1999, p. 259). Si bien la existencia de estas determinaciones puede constatarse claramente en los elementos corpóreos de la realidad, para Agustín es claro que estos principios están presentes también en el espíritu de los hombres, pues así como el resto de la Creación, ellos buscan el lugar que les es propio, aquel donde encontrarán reposo, que en su caso concreto está determinado por su amor. El lugar propio del hombre está con el Creador, por ello su amor debería estar orientado a Él. Sin embargo, es claro que el hombre no siempre orienta su amor a Dios, y esto sucede a causa del pecado, entendido como una voluntaria dirección errónea del amor o, en otras palabras, una voluntad desordenada.

Todo en la Creación es naturalmente bueno, en la medida en que fue creado por Dios, que no solo es todopoderoso, sino también bondad suprema. Al aceptar esto, Agustín afirma también que el mal no existe, pues su existencia sería incompatible con la de un ser como Dios. Sin embargo, ¿cómo explicar y justificar que algo que no existe ejerza una presencia tan poderosa en el mundo? Para Agustín el mal no es una realidad positiva, sino una carencia, es la privación del bien; así, en la medida en que todo lo creado es bueno en algún grado, lo que carece de bondad carece de sustancia y de existencia. El mal no es una sustancia, porque si fuera tal sería bueno y, como lo establece el mismo Agustín, "no hay naturaleza que sea mala, y este nombre de malo no denota otra cosa que la privación de lo bueno" (Agustín, civ. Dei., 11, 22). Queda una pregunta: ¿cuál es el origen del mal, si no está en Dios ni en un principio del mal? La respuesta es clara: el origen del mal está en la criatura, concretamente en el hombre y en su voluntad desordenada, que en el ejercicio de su libertad decide 
apartase de Dios. Es importante señalar dos cosas: primero, que el mal proviene de una elección equivocada y no de un objeto, y segundo, que esta elección no implica que el hombre escape del orden establecido, sino que invierte el orden de los fines, es decir, busca un orden erróneo, por lo que es posible definir el mal como una corrupción del orden original de la Creación.

Ahora bien, para Agustín el hombre naturalmente desea ser feliz, y el deseo de alcanzar la felicidad es lo que lo impulsa a vivir. Pero dicha felicidad no es posible sin paz, esto es, sin un estado de ausencia de disensiones y conflictos. La paz es uno de los mayores bienes, tanto de la vida eterna como de la temporal; sin embargo, solo en la primera se presenta en forma plena, pues es necesario un estado de unidad, de verdadera y absoluta justicia, y la disposición total de los corazones hacia Dios, para poder gozar del estado de plenitud que resulta del disfrute de la visión del Creador. Sabiendo qué es la paz perfecta, podrá determinarse la relación que la paz imperfecta - aquella de la que disfruta el hombre mientras está en la Tierra - guarda con la perfecta. Agustín define la paz como la tranquilidad del orden, y el orden no es otra cosa que la disposición de cosas iguales y desiguales, cada una en su propio lugar (civ. Dei, 19, 13), por esto la paz es el estado propio de la Ciudad Celestial; solo allí gobernará Dios con verdadera y absoluta justicia; solo allí los corazones de los hombres se orientarán plenamente a Dios y existirá una perfecta concordia entre la criatura y el Creador.

Por naturaleza, el hombre hace parte del orden perfecto, y por esto gozó inicialmente de la paz absoluta; pero debido a que la irrupción del pecado incide en todos los órdenes de la realidad, el hombre pasó a hacer parte de un estado de desorden, de perversidad, de aversión hacia el Creador y de conversión hacia las criaturas inferiores. ¿Qué pasa entonces con el hombre?, ¿está acaso condenado a una situación permanente de desorden?, ¿no goza de ninguna manera de paz o tranquilidad?, ¿no puede ser feliz?

A pesar de la situación trastornada de la humanidad, esta conserva hasta cierto punto el orden natural, pues de la misma manera que el resto de la Creación, está dispuesta según medida, número y peso, esto es, según el modelo universal establecido desde el origen. Este orden tiene un único y absoluto creador: Dios, que

[...] con su eterna sabiduría creó todas las naturalezas y justísimamente las dispone y ordena, y como más excelente entre todas las cosas terrenas, formó el linaje mortal de los hombres [...] les repartió la paz temporal, de la manera que la puede haber en la vida mortal; y esta paz se la dio al hombre en la misma salud, incolumidad y comunicación de su especie; y le dio todo lo que es necesario tanto para conservar, como para adquirir esta paz. (Agustín, civ. Dei., 19, 13)

La paz en el ámbito temporal se convierte en un bien dudoso e incierto; pese a esto, es un bien tan singular, que "aun en las cosas terrenas y mortales no solemos oír cosa de mayor gusto, ni desear objeto más agradable, ni, finalmente, podemos 
hallar cosa mejor" (Agustín, civ. Dei, 19, 11).Ejemplo de esto son aquellos que desean la guerra, aun cuando desean vencer y, así, alcanzar la gloriosa paz, o aquellos que con malicia y codicia turban la tranquilidad de otros, mientras que en su hogar, con su mujer y sus hijos, luchan porque exista siempre paz. Pero de no existir algún tipo de paz en la vida terrena, sería imposible la existencia y el progreso de la humanidad.

En este sentido, podemos decir que para el hombre resulta casi imposible alcanzar la plenitud de la felicidad, especialmente si consideramos que su logro no depende de cada hombre, sino del conjunto de ellos. En el ámbito individual, el hombre tiene que hacer frente a circunstancias difíciles de tolerar, pero los problemas que supone la vida en comunidad y las relaciones con otros no constituyen un mal menor. Las relaciones sociales generan inquietud en el corazón del ser humano. El no poder tener certeza de los demás, pero tampoco tener pleno dominio sobre las situaciones cotidianas que involucran a los seres queridos, produce dolor y sufrimiento; nos dirá el mismo Agustín que

no hay traición más secreta y oculta que la que se encubrió bajo el velo de oficio o bajo algún pretexto de amistad sincera. Porque fácilmente te podrás precaver y guardar del que es enemigo declarado, pero este mal oculto, intestino y doméstico, no sólo existe, sino que también le mortifica antes que pueda descubrirle. (Agustín, civ. Dei., 19, 3)

Si la vida en el ámbito doméstico es compleja, la esperanza en la paz y la felicidad se perderá en espacios como el de la ciudad o el de la comunidad universal, en los que no solo las relaciones son más enmarañadas, sino que la discordia y las disensiones entre los hombres son mayores.

Como vimos, Agustín afirma la existencia de un cierto orden en la vida del hombre, pues de lo contrario, la vida en la Tierra sería impensable, tanto en el ámbito individual como comunitario. Aunque ese orden es relativo, supone también un amor bien ordenado, armonía interna y externa, tanto en el cuerpo como en el alma, y entre uno y otra. Finalmente, en la medida en que el hombre es un ser social, es necesaria la ordenada concordia entre unos y otros, lo cual no depende solo del hombre, pues este no puede llegar a dicho estado sin la ayuda del Creador y sin estar con Él, pues

[...] toda paz, ya sea la del cuerpo, ya la del alma, o juntamente la del cuerpo y del alma, la refiere a aquella paz que tiene el hombre mortal con Dios inmortal, de modo que tenga la ordenada obediencia en la fe bajo la ley eterna. (Agustín, civ.Dei., 19, 13)

Para Agustín, el hombre es un ser social por naturaleza, y establece que tal condición, aunque resulta problemática, también ofrece ciertas ventajas, pues los grupos de hombres más o menos determinados y organizados no solo facilitan la satisfacción de necesidades primarias, como el abastecimiento de alimento o el comercio, sino que lo llevan a buscar, de forma permanente, mecanismos 
que le permitan vivir con sus semejantes de la mejor manera posible, esto es, conservando la paz y la concordia. Así, "de la misma manera que la vida de los santos, la vida del sabio y del hombre en general es social, pues ésta es la única forma en que es posible el progreso y el logro de ciertos fines" (Agustín, civ.Dei., 19, 5).

La sociedad es pues una dimensión esencial, tanto de la vida terrena como de la vida celestial. Sobre esto, Agustín identificará tres espacios en los que el hombre se desarrolla como ser social: la familia, la ciudad o república y, finalmente, el orbe de la tierra, que también denomina comunidad universal. A cada una de estas agrupaciones humanas corresponde un grado de la política, pero es la ciudad o república la que permite mostrar, en términos generales, cómo su dinámica social y política constituye un elemento determinante para la vida del hombre en el mundo.

El examen agustiniano de la república resulta de particular importancia, no solo porque parte de la exposición ciceroniana de aquella, sino porque hace evidente la manera en que Agustín comprende al hombre en el marco de su dimensión social. Es importante tener en cuenta que las expresiones ciudad o república no se emplean en un sentido estrictamente político o en cuanto espacios físicos, sino en sentido moral y social; tales expresiones hacen referencia, más bien, a la ciudadanía, es decir, a una colectividad de hombres que viven en comunión. Para Agustín este hecho no viene dado por hacer parte de esta o aquella colectividad, de esta o aquella población. Nacer o vivir en un lugar específico, estar o no de acuerdo con la forma de vida y las creencias de una comunidad son determinaciones que pueden cambiar eventualmente, pues esto hace parte de la dinámica propia del hombre caído. Por tal razón, para Agustín, estos no son hechos decisivos a la hora de determinar una ciudadanía. Ser ciudadano depende de algo mucho más fundamental: el amor.

Cicerón define la república como "cosa que pertenece al pueblo” (1991, pp. 25, 39), y precisa que pueblo no es todo conjunto de hombres reunido de cualquier manera, sino el conjunto de una multitud asociada por un mismo derecho que sirve a todos por igual. Luego de un cuidadoso examen, Agustín concluirá que de acuerdo con la formulación del Arpinate, en Roma nunca existió verdadera justicia, y por esto, tampoco una congregación de hombres asociada por un derecho común, pues sin justicia no es posible la existencia del derecho ni, por consiguiente, de un pueblo. Es claro que la lectura que hace Agustín de la definición ciceroniana se centra en la noción de justicia, y por ello, el objetivo es mostrar que esta nunca existió en la República romana, tal como la entendió el orador. Así, como lo señala O’Daly (2004), el interés de Agustín va más allá de esto y la exposición sirve a otros objetivos.

El primero de dichos propósitos es examinar cuáles serían las implicaciones de la justicia al servicio de Dios, esto es, la distinción entre lo que constituye la justicia para los cristianos y la realidad de la República romana cuando se rinde al culto de los falsos dioses (Agustín, civ.Dei., 19, 21). El segundo consiste en proponer 
una definición alterna, que considere el elemento cristiano y que justifique por qué solo puede denominarse verdadera república a la ciudad de Dios. En tercer lugar, Agustín, de acuerdo con el plan de la obra (la Ciudad de Dios), hará frente a las acusaciones paganas, según las cuales el cristianismo prepara la ruina de la República, puesto que le enseña al hombre a renunciar al mundo y, por lo tanto, aparta al ciudadano del servicio al Imperio. Finalmente, Agustín se servirá de la definición de Cicerón para mostrar que, pese a la situación trastornada de la humanidad, esta goza de cierta paz y tranquilidad, y ello sucede gracias a las dinámicas públicas y políticas que son propias de las naciones.

Aunque existen distintos elementos importantes en torno del análisis que Agustín hace de la definición ciceroniana, resulta de particular interés el giro que presenta la noción de pueblo, pues aunque Agustín está de acuerdo con Cicerón en que dicha noción supone una multitud de hombres, dista del Arpinate en que, para este, el fundamento del pueblo es la ley y el derecho, mientras que para Agustín lo es el amor común de bienes realmente idénticos. Agustín replantea la definición y establece que un pueblo "es una congregación de muchas personas, unidas entre sí por la comunión y conformidad de los objetos que ama" (civ. Dei., 19, 24). De esta manera, un pueblo es realmente tal cuando en él existe la concordia de las razones y de los corazones que liga el conjunto de los individuos y de las personas; de este modo, podemos afirmar con Gilson (1954) que un pueblo se constituye cuando su vínculo es la concordia. La nueva definición le permite a Agustín reconocer comunidades que puedan denominarse pueblos, a pesar de que en ellas no impere la justicia, como en el caso de Roma; además, al llevar la definición de lo jurídico a lo que podemos denominar comunitario o vital, el énfasis se pone en el amor, que en el pensamiento agustiniano revierte particular importancia, pues es la potencia fundamental de la vida en cuanto es fuente de concordia y unión: "dos amores fundaron dos ciudades; la terrena, el amor propio hasta llegar a menospreciar a Dios, y la celestial, el amor a Dios, hasta llegar al desprecio de sí propio" (civ.Dei., 14, 28).

Notamos que en este punto Agustín se distancia del espíritu jurídico romano y rechaza la idea de que la ley humana sea la absoluta rectora de la vida del hombre. Sin embargo, es preciso señalar que con lo anterior Agustín no desconoce que el derecho tenga lugar en la República, tampoco que sea útil a los hombres, pues reconoce que las normas $-\mathrm{y}$ en su caso concreto, el derecho romano - posibilitan el gobierno humano y la vida en comunidad. Es necesario que el hombre asuma un compromiso social y de responsabilidad política, pues solo así se generan las condiciones para el ejercicio de la virtud, la justicia y la concordia, que son finalmente las que mantienen la paz terrena. En este sentido podemos decir que para Agustín, la República y el pueblo se determinan por circunstancias concretas y particulares, por la experiencia, por las cosas que aman - aunque estas sean bienes materiales con valores temporales - , y no por el deber ser o la ley humana.

Para Agustín el ejercicio del poder político no es una condición natural en el hombre porque este no fue creado para dominar a sus semejantes, sino a las 
bestias. La situación de dominio, de unos hombres sobre otros, está dada por su condición pecadora, por su soberbia y por su afán de alcanzar una condición similar a la de Dios, es decir, como creador, ordenador y cuya potestad es suprema. Tanto para la paz eterna como para la temporal, es necesaria la dinámica entre el mando y la obediencia; sin embargo, solo en la primera, donde gobierna Dios, este ejercicio es perfecto y justo; en la segunda, donde los hombres gobiernan sobre los hombres, la relación termina siendo de servidumbre, como consecuencia no de la naturaleza, sino del pecado original:

[...] la primera causa de la servidumbre es el pecado; que se sujete el hombre uno a otro con el vínculo de la condición servil, lo cual no sucede sin especial providencia y justo juicio de Dios, en quien no hay injusticia y sabe repartir diferentes penas conforme a los méritos de las culpas. (civ.Dei., 19, 15)

En la ciudad terrena se establecen relaciones sociales de jerarquía y de coerción que garantizan algún tipo de orden entre los hombres. Esta coerción no es contraria al derecho, sino conforme con él; se instaura luego de la caída del hombre, y por eso se vincula con la falta y la culpa. Este derecho garantiza la convivencia humana en sociedad, en la medida en que regula las conductas externas; sin embargo, no puede garantizar la verdadera justicia, pues no puede "legislar en el foro interno de las personas" (Boron et al., 2000). A este respecto es importante señalar que Agustín juzga positivamente este tipo de derecho, pues genera condiciones de civilidad; de ahí que enfatice el respeto a la autoridad pagana - que se representa en el cuerpo del derecho romano-. "De este derecho y de su aceptación resulta que pueda haber garantías de paz terrenal. Paz que es aprovechable tanto por paganos como cristianos" (2000), tanto por ciudadanos de este mundo, como por los peregrinos de él.

Es importante señalar que el hombre es un ser racional; sin embargo, no solo se mueve por razones, sino también por emociones. Agustín comprende al ser humano en términos de deseos, de aspiraciones, de anhelos. Lo que los hombres anhelan es justamente lo que los define. Este deseo puede entenderse como la necesidad que está presente en su naturaleza incompleta y, asimismo, como la totalidad de la experiencia, que se nos presenta como inestable, corruptible y desordenada. Sin embargo, Agustín reconoce también que a pesar de la situación trastornada y corrupta en la que está inmersa la humanidad, existen ciertos elementos, como la sociedad y la política, que, hasta cierto punto, pueden mantener y preservar el orden y la paz en el marco de la regulación de los deseos, las voluntades y los espíritus de los hombres.

Como lo señalamos anteriormente, el hombre es un ser social, pero no es sencillo imponer un vínculo social entre los hombres, que son tan diversos y tan inconstantes. Para aplicarles reglas comunes; para plegarlos ante el mando y hacer que la obediencia se acepte; para hacer que la pasión ceda a la razón, y la razón individual a la razón pública, hace falta algo más fuerte que la fuerza material, más respetable que el interés, más seguro que una teoría filosófica, 
más estable que un convenio; algo, en suma, que resida por igual en el fondo de todos los corazones y que radique allí con imperio (De Coulanges, 2002, p. 149). Este algo es lo que Agustín denomina concordia, que es una especie de armonía, de simpatía entre los hombres, generada por la comunidad en torno a un objeto. La concordia es lo que permite que entre los hombres se dé un acuerdo de voluntades y, de esta manera, un verdadero pueblo y no simplemente la unión de una multitud en una sumisión común.

Finalmente, hay que decir que Agustín reconoce la necesidad de un elemento adicional al amor, que regule las relaciones entre los hombres y determine los caminos por los cuales la humanidad pueda acceder a un cierto bienestar; sabe también que por el pecado original la vida del hombre supone una serie de problemas, como la necesidad de afirmar el dominio frente a sus semejantes y la incapacidad de vivir según el dictamen de su razón. Por esto, Agustín ve la necesidad de recurrir a medios coactivos para regular la vida de los hombres en la Tierra y así, a largo plazo, alcanzar la paz; sin embargo, esto solo es posible mediante la persuasión no coactiva, pues para conseguir dicho objetivo es necesario el libre consentimiento de la voluntad, así como de un acuerdo y un compromiso entre voluntades. En esto radica la importancia de la definición de pueblo que Agustín nos ofrece, pues supone, justamente, un acuerdo de voluntades y un fin último común, a la vez que indica la necesidad de organizaciones y órdenes sociales y políticos que estén en función de orientar dichas voluntades, por distintos medios, hacia el bien común.

\section{$\mathbf{R}_{\text {eferencias }}$}

Agustín, San. (1946). De ord. El orden. Madrid: Biblioteca de Autores Cristianos. [Edición de Victorino Capánaga].

Agustín, San. (1951). Ep. Cartas. Madrid: Biblioteca de Autores Cristianos. [Edición de Lope Cilleruelo].

Agustín, San. (1957). Gn.litt. Comentario literal al Génesis. Madrid: Biblioteca de Autores Cristianos. [Edición de Balbino Martín].

Agustín, San. (1995). Retr. Las retractaciones. Madrid: Biblioteca de Autores Cristianos. [Edición de Teodoro C. Madrid].

Agustín, San. (1988). De civ. Dei. La ciudad de Dios. Madrid: Biblioteca de Autores Cristianos. [Edición de Victorino Capánaga].

Agustín, San. (2001). Confess. Confesiones. México: Porrúa. [Edición de Francisco Montes].

Bermejo, F. (2008). El maniqueísmo: estudio introductorio. Madrid: Trotta. 
Borón, A. et al. (2000). La filosofía política clásica: de la Antigüedad al Renacimiento. Buenos Aires: Consejo Latinoamericano de Ciencias Sociales.

Cicerón, M. (1991) Sobre la República. Madrid: Gredos. [Edición de Álvaro D’Ors].

Deane, H. (1963). The political and social ideas of St. Augustine. Nueva York: Columbia University Press.

De Coulanges, F. (2002) La ciudad Antigua. Bogotá: Panamericana. [Edición de M. Cigés Aparicio].

Flórez, A. (2004). San Agustín. La persuasión de Dios. Bogotá: Panamericana.

Gilson, E. (1954). Las metamorfosis de la ciudad de Dios. Buenos Aires: Troquel SRL. [Edición de Benjamín Agüero].

Harrison, C. (2000). Augustine. Christian truth and fractured humanity. Nueva York: Oxford University Press.

Mas, S. (2006). Pensamiento romano. Una historia de la filosofía en Roma. Valencia: Tirant lo Blanch.

Moreno, J. (1999). San Agustín: el orden en Dios y en la naturaleza. Espíritu. Cuadernos del Instituto Filosófico de Balmesiana, (48), 255-262.

O'Daly, G. (2004). Augustine's city of God. A Reader's Guide. Nueva York: Oxford University Press.

TeSelle, E. (1998). Living in two cities: Augustinian trajectories in political thought: Scranton: University of Scranton Press.

Von Heyking, J. (2001). Augustine and politics as longing in the world. Missouri: University of Missouri Press. 